\title{
SENSIBILIDADE MORAL DOS ENFERMEIROS AVALIADA POR SCOPING REVIEW*
}

\author{
Carlise Rigon Dalla Nora ${ }^{1}$, Elma Zoboli², Margarida Maria Vieira ${ }^{3}$
}

\begin{abstract}
RESUMO: Esse estudo tem como objetivo identificar os fatores que influenciam a sensibilidade moral dos enfermeiros nos serviços de saúde, a partir de publicações sobre a temática. Para tanto, realizou-se uma scoping review no período de agosto a dezembro de 2015 em bases de dados da literatura em saúde. Foram incluídos artigos teóricos e originais realizados com estudantes de Enfermagem e equipes de saúde com enfermeiros, publicados em inglês, espanhol e português. A amostra foi de 28 estudos. Da análise, resultaram duas categorias: "fatores pessoais" e "fatores contextuais" da sensibilidade moral. Conclui-se que o fator que tem maior peso na formação/expressão da sensibilidade moral dos enfermeiros são os "fatores pessoais". A sensibilidade moral tem uma natureza multidimensional, e que precisa ser complementada com uma preparação do enfermeiro para identificar e lidar de forma responsável e prudente com os problemas éticos da prática.
\end{abstract}

DESCRITORES: Moral; Desenvolvimento moral; Ética de enfermagem; Enfermagem.

\section{MORAL SENSITIVITY OF NURSES ASSESSED THROUGH SCOPING REVIEW}

\begin{abstract}
This study aims to identify the factors that influence the moral sensitivity of nurses in health services, based on publications on the theme. Therefore, we conducted a scoping review for the August-December 2015 period in health literature databases. Theoretical and original studies with nursing students and health teams with nurses, published in English, Spanish and Portuguese, were included. The sample consisted of 28 studies. Two categories emerged from the analysis: "personal factors" and "contextual factors" of moral sensitivity. We concluded that "personal factors" have the greatest impact on the formation/expression of moral sensitivity of nurses. Moral sensibility has a multidimensional nature and must be complemented with nurse's training to identify and deal responsibly and prudently with the ethical problems of clinical practice.

DESCRIPTORS: Moral; Moral development; Nursing ethics; Nursing.
\end{abstract}

\section{SENSIBILIDAD MORAL DE LOS ENFERMEROS EVALUADA POR SCOPING REVIEW}

RESUMEN: Estudio cuyo objetivo fue identificar los factores que influyen en la sensibilidad moral de los enfermeros en los servicios de salud, a partir de publicaciones sobre la temática. Para ello, se realizó una scoping review entre agosto y diciembre de 2015, en bases de datos de literatura en salud. Fueron incluidos artículos teóricos y originales realizados con estudiantes de Enfermería y equipos de salud con enfermeros, publicados en inglés, español y portugués. La muestra incluyó 28 estudios. Del análisis surgieron dos categorías: "factores personales" $y$ "factores conceptuales" de la sensibilidad moral. Se concluye en que el factor de mayor peso en la formación/expresión de la sensibilidad moral de los enfermeros son los "factores personales". La sensibilidad moral tiene naturaleza multidimensional, que necesita complementarse con preparación del enfermero para identificar y enfrentar de manera responsable y prudente los problemas éticos de la práctica.

DESCRIPTORES: Moral; Desarrollo Moral; Ética en Enfermería; Enfermería.

\footnotetext{
*Artigo extraído da tese intitulada: "Sensibilidade moral dos enfermeiros face aos problemas éticos na atenção primária à saúde". Universidade Católica Portuguesa, 2017.

${ }^{1}$ Enfermeira. Doutoranda em Enfermagem pela Universidade Católica Portuguesa. Porto, Portugal.

${ }^{2}$ Enfermeira. Pós-doutora em Bioética. Doutora em Saúde Pública. Docente de Enfermagem da Universidade de São Paulo. São Paulo, SP, Brasil.

${ }^{3}$ Enfermeira. Doutora em Filosofia. Docente da Universidade Católica Portuguesa. Porto, Portugal.
}

Autor Correspondente:

Carlise Rigon Dalla Nora

Universidade Católica Portuguesa.

R. Arquiteto Lobao Vital, Apartado 2511 - 4202-401 - Porto, Portugal

E-mail: carliserdn@gmail.com
Recebido: $15 / 06 / 2016$

Finalizado: 16/03/2017 


\section{- INTRODUÇÃO}

Os inúmeros avanços na tecnologia de saúde têm levado ao aumento e ao surgimento de novos problemas éticos, ainda mais complexos e significativos para a prática dos enfermeiros ${ }^{(1-2)}$. Esses problemas são relativos a aspectos, questões ou implicações éticas, de ocorrência comum ou não, na prática da atenção à saúde. A sensibilidade moral dos enfermeiros permite distinguir os problemas de natureza ética de outros problemas.

A sensibilidade moral também é denominada de "sensibilidade ética" e estudos ${ }^{(3)}$ referem a dificuldade de consenso na distinção entre estes termos. Para este estudo, a sensibilidade moral é definida como a compreensão contextual e intuitiva da situação de vulnerabilidade do paciente pelo enfermeiro, levando em consideração as consequências éticas das decisões tomadas em nome daquele $^{(4)}$. Para tomar decisões éticas, o enfermeiro precisa antes reconhecer, e depois ter e mostrar sensibilidade perante a situação de vulnerabilidade do paciente.

O desenvolvimento do quadro teórico deste estudo é derivado do conceito de sensibilidade moral, sendo este um fenômeno complexo que envolve mais do que conhecimento científico: incluiu também sensações, sentimentos, conhecimento moral e habilidades do sujeito, pois abrange o problema ético em situações particulares nas relações interpessoais entre pacientes e enfermeiros ${ }^{(5)}$. Para La Taille ${ }^{(6: 88)}$, a sensibilidade moral corresponde "a capacidade de perceber as dimensões morais de certas ações ou situações nas quais estas não aparecem com evidência". A falta de sensibilidade moral pode levar a ações imorais, ou no mínimo, inadequadas ${ }^{(6)}$.

As dimensões teóricas da sensibilidade moral descritas por Lützén ${ }^{(4)}$ envolvem: a orientação interpessoal, a estruturação do significado moral, a benevolência, a autonomia, o conflito moral,confiança do profissional no seu próprio conhecimento e regras. A sensibilidade moral, portanto, envolve a capacidade de lidar com problemas éticos e isso, dependendo das circunstâncias, pode tornar-se uma responsabilidade pesada para o enfermeiro, demandando resistência e força moral excepcionais.

É fundamental identificar os fatores que influenciam a sensibilidade moral dos enfermeiros, a fim de que seja possível criar estratégias para sua promoção, melhorando a sua conduta profissional, ou seja, a sua capacidade de tomada de decisão ética, e por consequência, a sua satisfação profissional e a qualidade do atendimento prestado.

Este artigo faz parte de um estudo de doutoramento em Enfermagem que pesquisou a sensibilidade moral de enfermeiros na Atenção Primária em Saúde (APS) de Portugal e Brasil. Aqui, apresentase a etapa de revisão da literatura que teve como objetivo identificar os fatores que influenciam a sensibilidade moral dos enfermeiros nos serviços de saúde.

\section{- MÉTODO}

A metodologia de scoping review, descrita inicialmente por Arksey e $\mathrm{O}^{\prime}$ Malley ${ }^{(7)}$, e posteriormente sistematizada por Levac, Colquhoun e $\mathrm{O}^{\prime} \mathrm{Brien}^{(8)}$, foi a adotada para a realização deste trabalho. Ela visa à obtenção de resultados amplos e abrangentes e com menor profundidade sobre um dado tema, embora compartilhe algumas características da metodologia da revisão sistemática, como ter um desenho metódico, transparente e replicável ${ }^{(9)}$. A principal diferença entre a revisão sistemática e a scoping são: a questão inicial é ampla, aberta, em vez de focada como na revisão sistemática; critérios de inclusão/exclusão podem ser estabelecidos a posteriori, em vez de antes de começar a revisão; a escolha dos estudos não foca na qualidade da investigação; pode ou não envolver extração de dados; a síntese é fundamentalmente qualitativa e raramente quantitativa; é utilizada para identificar as variáveis e as lacunas existente na literatura ${ }^{(10)}$.

Foram utilizados os seguintes passos: identificação da questão de pesquisa; identificação de estudos relevantes; seleção dos estudos; extração de dados; separação, sumarização e relatório de resultados; e comunicação dos resultados ${ }^{(7-8)}$.

A questão de pesquisa deste estudo, conforme exposto anteriormente, foi: "quais os fatores que influenciam a sensibilidade moral dos enfermeiros nos serviços de saúde?" Para a busca ser abrangente, 
diferentes fontes foram consultadas, através das plataformas BVS (Biblioteca Virtual da Saúde), EBSCO (EBSCOhost Online Research Databases) e Web of Science, as quais abrangem as bases: Lilacs (Literatura Latino-Americana e do Caribe em Ciências da Saúde), Ibecs (Índice Bibliográfico Espanhol de Ciências da Saúde), Medline (Medical Literature Analysis and Retrieval System Online), Biblioteca Cochrane, SciELO (Scientific Electronic Library Online), BDENF (Base de Dados de Enfermagem), CINAHL (Cumulative Index to Nursing and Allied Health Literature), e a ferramenta Google Scholar.

Utilizaram-se os seguintes descritores/MeSH e palavras-chave de assunto em inglês, espanhol e português: moral sensitivity, moral sensibility, ethical sensitivity, ethical sensibility, moral development, moral dilemma, ethical dilemma, ethics, nurs*, health services, health care. Foram realizadas as seguintes associações em inglês, com os operadores booleanos "and" e "or": moral sensitivity and nurs*, moral sensibility and nurs*, ethical sensitivity and nurs*, ethical sensibility and nurs*, moral sensitivity and moral dilemma, moral sensitivity and ethical dilemma, ethical sensitivity and moral dilemma and ethics, moral sensitivity and nurs* and health services, moral sensitivity and health care, moral sensitivity or ethical sensitivity, moral sensitivity or moral development. As mesmas associações foram realizadas em português e espanhol. As referências bibliográficas dos trabalhos incluídos foram revisadas, a fim de encontrar mais algum artigo de interesse para este estudo.

Como a scoping review é exploratória, todos os resultados encontrados acerca do tema devem ser incluídos, assim, por ser mais inclusiva, permite que os pesquisadores identifiquem lacunas na investigação existente ${ }^{(10)}$. Para selecionar os estudos, foram utilizados os seguintes critérios de inclusão: artigos de pesquisa teóricos e originais, publicados em inglês, espanhol e português, realizados com estudantes de Enfermagem e equipes de saúde com enfermeiros. Não se estabeleceu um limite temporal na busca, pois se considera que a ética é um tema que perpassa a ciência desde sua criação.

Os critérios de exclusão foram: estudos realizados com usuários ou outros profissionais, equipes de saúde que não incluíssem enfermeiros, estudos com desenho de pesquisa ou objetivos pouco definidos ou pouco explicitados.

As buscas foram executadas entre os meses de agosto e dezembro de 2015, por dois pesquisadores, separadamente. Em fase posterior, compararam-se os resultados e resolveram-se as discordâncias por consenso. Em outras palavras: os pesquisadores confrontaram os resultados das buscas realizadas, independentemente um do outro, nas bases de dados, verificando as diferenças dos achados e sempre visando incluir o maior número de estudos possível. A Figura 1 exibe o processo de busca, de exclusão e de seleção dos estudos encontrados:

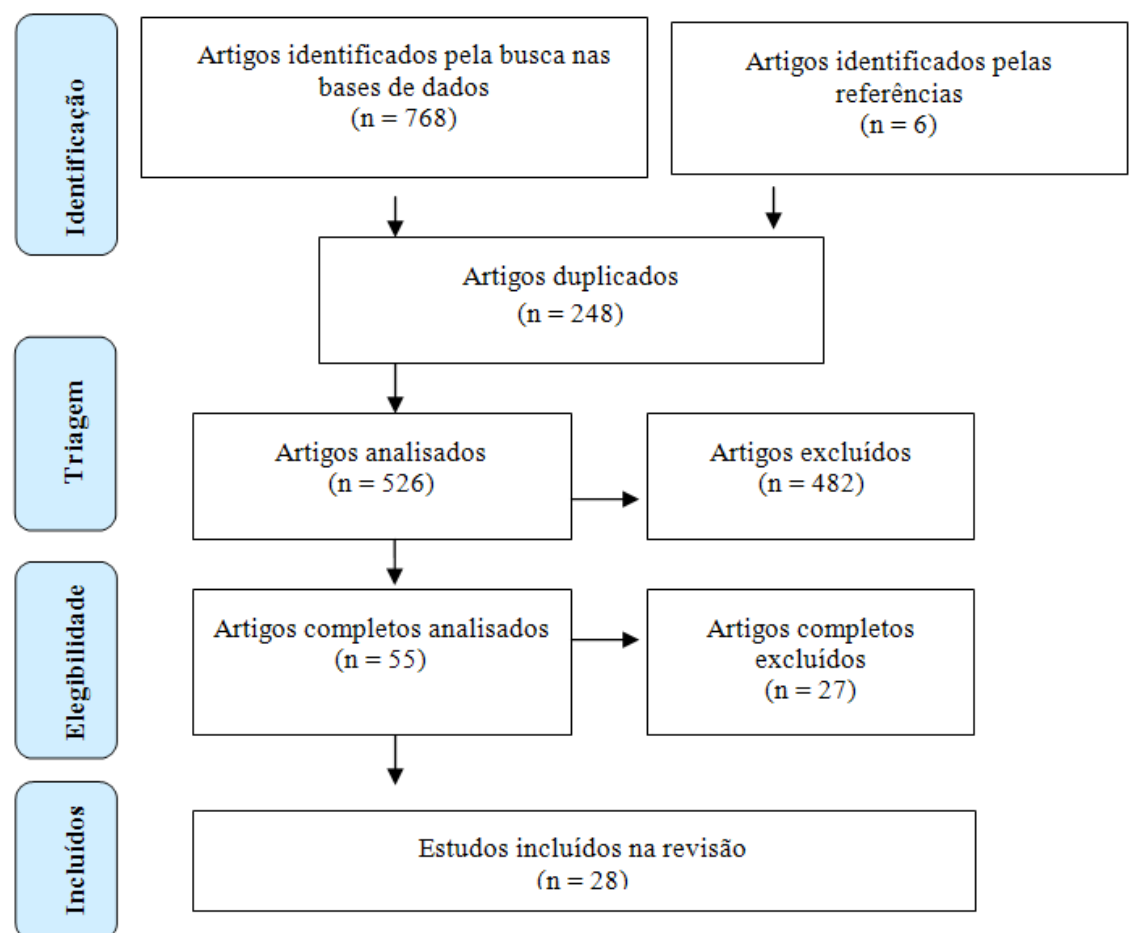

Figura 1 - Seleção dos estudos nas bases de dados. Porto, Portugal, 2016 
Para a etapa de separação, sumarização e relatório dos elementos essenciais encontrados em cada estudo, foi utilizado um instrumento estruturado próprio. Esse instrumento permitiu a síntese, interpretação dos dados e a análise numérica básica da extensão, da natureza e da distribuição dos estudos incorporados na revisão. Itens como ano de publicação, periódico, local de realização do estudo, tipo de serviço e metodologia utilizada, foram agrupados.

$\mathrm{Na}$ etapa final, foi realizada a compilação e comunicação dos resultados, com a intenção de apresentar a visão geral de todo o material, através de uma construção temática, organizada de acordo com a natureza dos fatores que influenciam a sensibilidade moral dos enfermeiros.

\section{RESULTADOS}

Foram identificadas 774 publicações sobre o tema, das quais 28 foram selecionadas para integrar a amostra, sendo esta composta por 19 estudos originais ${ }^{(1-2,4-5,11-25)}$ e nove estudos teóricos ${ }^{(26-34)}$. Todos os estudos incluídos estavam publicados em inglês.

Onze estudos (40\%) foram publicados na revista Nursing Ethics (fator de impacto 1.247, avaliação ano 2015), dois (8\%) no Journal of Advanced Nursing (fator de impacto 1.741, avaliação ano 2015) e dois (8\%) na Nursing Philosophy (fator de impacto 0.833, avaliação ano 2015); os demais estudos foram publicados em diferentes revistas da área da saúde.

Considerando a classificação que considera sete níveis de qualidade das evidências científicas, os resultados desta revisão indicaram evidências de nível seis (derivadas de um único estudo descritivo ou qualitativo) e de nível cinco (evidências originárias de revisão sistemática de estudos descritivos e

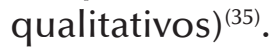

Quanto ao tema, 16 estudos abordavam a sensibilidade moral|(2,4-5,11-14,16,19,21-22,24-25,27-29) e 12 estudos abordavam a sensibilidade ética ${ }^{(1,15,17-18,20,23,26,30-34)}$. Quanto ao ano de publicação, os estudos selecionados distribuíram-se entre 2000 e 2015 (Figura 2).

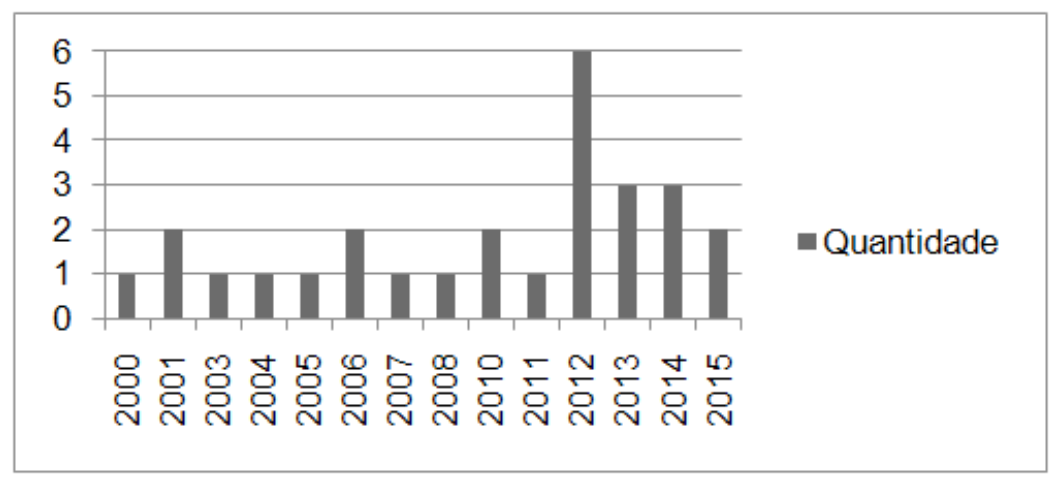

Figura 2 - Ano de publicação dos estudos. Porto, Portugal, 2016

Seis estudos foram realizados na Coreia ${ }^{(1-2,11,14,17,22)}$; quatro na Suécia ${ }^{(4-5,12-13)}$ e na Turquia( ${ }^{(15,20,24-25)}$; dois no Irã ${ }^{(19,23)}$; um na Espanha ${ }^{(18)}$, Estados Unidos ${ }^{(21)}$, Japão e Noruega ${ }^{(16)}$.

Oito estudos ocorreram em contexto hospitalar ${ }^{(4,11,14-17,19-20)}$; sete em Universidades ${ }^{(1-2,21-25)}$; dois em serviços de saúde mental ${ }^{(12-13)}$ e um em serviços de cuidados de saúde primários e hospitalar ${ }^{(18)}$.

Os participantes dos estudos totalizaram 2.013 enfermeiros ${ }^{(11-14,16-20)} ; 722$ estudantes de Enfermagem ${ }^{(1-2,21-25)} ; 778$ médicos $^{(12)}$ e 278 profissionais da saúde que incluíam enfermeiros, médicos e auxiliares de enfermagem ${ }^{(5)}$.

Dezenove estudos $(68 \%)$ derivaram de pesquisas quantitativas ${ }^{(1-2,4-5,11-22,24-25,27)}$ e nove de pesquisas qualitativas $(32 \%)^{(23,26,28-34)}$. Para a coleta de dados, os estudos utilizaram questionário ${ }^{(1-2,4-5,11-22)}$, buscas na literatura ${ }^{(26-28,30-31,33)}$ e entrevistas e grupos focais ${ }^{(23)}$. 
Quanto ao método de análise utilizado pelos estudos, 16 usaram a análise estatística ${ }^{(1-2,4-5,11-17,19-22,27),}$ quatro utilizaram análise de conteúdo ${ }^{(23,26,28,31)}$, um de análise fenomenológica ${ }^{(33)}$, um de análise pragmática ${ }^{(30)}$ e um de análise $\operatorname{Rasch}^{(18)}$.

Ao final, chegou-se a duas categorias para a comunicação dos resultados: "fatores pessoais" e "fatores contextuais" que interferem na sensibilidade moral dos enfermeiros.

\section{"Fatores pessoais" da sensibilidade moral}

Estes se referem aos fatores individuais que cada enfermeiro necessariamente apresenta e que, de alguma forma, influenciam a sua sensibilidade moral. Destaca-se na literatura a percepção do conflito moral $^{(2,11,14,16,19,21-22,26,30-31)}$; benevolência ${ }^{(2,12,14,19,21-22,30-31)}$; afetividade ${ }^{(1,23-26,29-31,34)}$; responsabilidade ${ }^{(4,11,14,17,22,30-31)}$; valores éticos e morais ${ }^{(2,4,16-17,31,33)}$; gênero $^{(12-13,22-23,26-27)}$; idade $^{(17,20,22)}$; e religião $\mathrm{O}^{(22-23)}$. Outros fatores também foram identificados, como: $\operatorname{coragem}^{(4,26,30-31)}$; $\operatorname{compromisso}^{(16,19,32)}$; intuição ${ }^{(31)}$; $\operatorname{atitude}^{(31)}$; empatia( ${ }^{(23,32)}$; receptividade ${ }^{(31)}$; $\operatorname{altruísmo~}^{(30-31)}$, curiosidade $^{(30)}$; reflexão ${ }^{(31)}$; veracidade $^{(15)}$; intolerância a atos antiéticos ${ }^{(31)}$; consciência dos efeitos das ações ${ }^{(31)}$; desenvolvimento psicossocial e cognitivo moral do profissional ${ }^{(30)}$. Estes fatores são considerados de difícil identificação em estudos quantitativos que utilizam questionários como fonte de coleta de dados. Por isso, a necessidade de incluir tanto pesquisas qualitativas quanto teóricas na presente revisão.

\section{"Fatores contextuais" da sensibilidade moral}

Nesta categoria são descritos os fatores relacionados às circunstâncias externas ao indivíduo que influenciam a sensibilidade moral dos enfermeiros. Referem-se a circunstâncias que se constituem ou se formam nos processos de trabalho desenvolvidos nas instituições.

Os fatores que se destacam na literatura são: formação $\mathrm{O}^{(11,13,18,20,22,24,27,32)}$; prática profissional(11,13,17-19,20,23,30); o clima ético/moral das instituições ${ }^{(12-13,16,18-19,28-30,33)}$; as normas, regulamentos e códigos da profissão ${ }^{(5,11,14,16,19,22-23,31-32)}$; a demanda de trabalho; a falta de tempo ${ }^{(11,16,19,30)}$; a colaboração e apoio para a resolução dos problemas éticos ${ }^{(13,16,18,29)}$.

No âmbito do ensino, a literatura consultada apresentou fatores relacionados à formação ${ }^{(11,13,18,20,22,24,27,32)}$, ao conhecimento ético ${ }^{(1,18-19,31)}$, à educação ética ${ }^{(17,20,23-25)}$, à competência e/ou profissionalismo $^{(1,26,30-32)}$ e à estruturação do significado moral ${ }^{(2,11-12,19,21-22)}$, sendo estes aspectos que interferem no processo de reflexão pelo qual o enfermeiro estrutura o seu pensamento moral para tomar decisões e realizar ações éticas.

Quanto à prática profissional, os anos de experiência profissional ${ }^{(11,13,17-20,23,30)}$ constituem o fator que mais influencia a sensibilidade ética dos enfermeiros, seguido da capacidade de comunicação $\mathrm{O}^{(19,23,26,29-31)}$ e da visão do papel profissional $\left.\right|^{(2,11-12,17,23)}$ que os enfermeiros possuem.

A sensibilidade moral dos enfermeiros pode ficar prejudicada devido ao conjunto de valores da instituição. Os valores relacionados ao ambiente de trabalho, também conhecido como "o clima moral da instituição", podem influenciar de forma positiva ou negativa a moral dos enfermeiros. Nesse âmbito, pode haver conflitos entre os valores pessoais dos enfermeiros e os valores da organização onde ele trabalha ${ }^{(19)}$.

Destaca-se que um ambiente de trabalho inadequado está associado a efeitos negativos como: estresse $^{(13,16,19)}$, ansiedade ${ }^{(16,19)}$, privação do sono ${ }^{(13,16)}$, sofrimento moral ${ }^{(1,28)}$, burnout ${ }^{(28)}$, frustração ${ }^{(1)}$, insatisfação no trabalho ${ }^{(1,28)}$, diminuição na qualidade do atendimento ${ }^{(28)}$ e abandono da profissão ${ }^{(13)}$.

Por outro lado, um ambiente institucional pode ser considerado positivo e "ético", quando os valores da instituição estão explícitos e em coerência com aqueles dos enfermeiros. A instituição que possui valores claros e promove espaços para o compartilhamento de saberes, permite que os enfermeiros sintam-se seguros para serem ouvidos, promovendo uma maior satisfação no trabalho ${ }^{(28)}$.

As regras, regulamentos e códigos podem ser eficazes para melhorar a sensibilidade moral, pois contribuem definindo as ações que são esperadas de um enfermeiro. É necessário oferecer espaço 
para o enfermeiro falar e analisar os problemas éticos que aparecem na relação com o paciente, permitindo a identificação dos recursos institucionais de apoio de que necessitam ${ }^{(32)}$.

A partir do mapeamento da literatura, evidencia-se que os fatores que tem mais peso na formação/ expressão da sensibilidade moral são os "fatores pessoais", pois estes foram citados em maior quantidade (71 ocorrências) na literatura.

\section{DISCUSSÃO}

Esta revisão traz uma amostra consistente de estudos na área da sensibilidade moral de enfermeiros, publicados em revistas de qualidade científica com elevado fator de impacto. A pesquisa se mostrou abrangente, abarcando estudos dos últimos 15 anos. Destaca-se que nenhum estudo em português ou espanhol foi encontrado, demonstrando a carência de estudos sobre esse tema no Brasil e em outros países com aqueles idiomas. Porém, nos últimos quatro anos, os estudos sobre a sensibilidade moral têm se desenvolvido consideravelmente no âmbito internacional.

A scoping review permitiu verificar que os fatores que tem maior peso na formação/expressão da sensibilidade moral dos enfermeiros são os "fatores pessoais". Isso não significa que os "fatores contextuais" não tenham peso, mas sim, que ela é desenvolvida, em boa parte, pelas características pessoais dos profissionais. Estudo ${ }^{(31)}$ de revisão corrobora com os achados ao apresentar que os cinco domínios principais que caracterizam a sensibilidade ética são: cognitivo, afetivo, habilidade, responsabilidade e conhecimento. Todos estes domínios, com exceção do conhecimento, estão incluídos na categoria "fatores pessoais".

Outro estudo(4) $^{(4)}$ reforça os resultados, ao apontar que a sensibilidade moral é um "atributo pessoal" necessário para lidar com os problemas éticos quando os profissionais são confrontados com situações em que precisam tomar decisões pelo paciente, o qual está em uma condição vulnerável devido a sua doença. Nesse sentido, as qualidades pessoais são importantes no processo de tomada de decisão, pois o enfermeiro não pode basear sua decisão somente no código de ética ou normas, mas sim, precisa possuir sensibilidade moral para perceber a situação de vulnerabilidade do paciente ${ }^{(4)}$.

Uma revisão(36) apontou que os problemas éticos podem ocorrer em situações comuns da prática cotidiana e os enfermeiros precisam estar preparados e sensibilizados para reconhecer e enfrentar esses problemas com prudência. Estudo ${ }^{(37)}$ descreve que, inicialmente, o enfermeiro precisa perceber uma situação como causadora de problemas ético, e esse processo é resultado dos valores pessoais, provenientes da cultura, da família, do conhecimento profissional e dos valores adquiridos através da formação e da experiência profissional. No presente estudo, a percepção do conflito moral é um dos fatores que tem maior influência na sensibilidade moral dos enfermeiros.

De acordo com os achados, os fatores "contextuais" também influenciam a sensibilidade moral dos enfermeiros, porém em menor medida. Estudo ${ }^{(38)}$ refere que um ambiente de trabalho portador de um conjunto de valores que orientam a boa prestação de cuidados influencia positivamente as atitudes e comportamentos dos enfermeiros quanto à forma de lidar com os problemas éticos da sua prática. Ou seja, para a construção de relações respeitosas entre os profissionais, com vistas a compartilhar recursos de apoio para enfrentamento dos problemas, é fundamental que exista um ambiente de trabalho com algum nível de sensibilidade ética ${ }^{(38)}$.

Outros estudos $^{(39-41)}$ legitimam os achados desta revisão ao afirmar que os valores pessoais e organizacionais têm efeitos sobre a qualidade dos cuidados de enfermagem. De acordo com estudo ${ }^{(39)}$, dez principais valores éticos de Enfermagem são: dignidade humana, privacidade, justiça, autonomia na tomada de decisão, precisão e exatidão no cuidado, compromisso, relacionamento humano, simpatia, honestidade, competência individual e profissional.

Quando o enfermeiro não consegue atender os problemas éticos da sua prática, ele pode estar em risco de sofrimento moral ${ }^{(42)}$ e, sendo assim, o profissional também pode deixar de agir no melhor interesse do paciente. Por outro lado, quando o enfermeiro é eticamente competente, consegue lidar com os problemas de forma crítica, reflexiva e resolutiva. A razão pela qual o processo de trabalho pode influenciar na sensibilidade moral, é descrita em estudo ${ }^{(42)}$ que refere que a falta de tempo, a 
falta de apoio organizacional, os conflitos com outros profissionais, as políticas institucionais e os diferentes valores éticos e legais relacionados ao processo de tomada de decisão pelo enfermeiro podem deixar estes propensos a experimentar situações eticamente problemáticas.

Como principal achado desta revisão, destaca-se a natureza multifatorial da sensibilidade moral, o que permite questionar se é possível promovê-la no enfermeiro. Estudo ${ }^{(37)}$ aponta que sim, pois o desenvolvimento moral dos estudantes de Enfermagem precisa ser estimulado através de estratégias educativas, capazes de aumentar a sua capacidade de refletir e de questionar as decisões relativas ao cuidado, de forma a não limitar a dimensão ética dessas decisões. Outro estudo ${ }^{(43)}$ também corrobora com essa afirmação, ao entender que, da mesma forma como se desenvolvem habilidades clínicas, também as habilidades éticas e morais podem ser aprendidas por experiências e melhoradas e aperfeiçoadas ao longo do tempo, por meio de estratégias que incluem: educação ética permanente, grupo de discussão, trato com comitês de ética com enfermeiros e/ou equipe interdisciplinar. Para a promoção da sensibilidade moral, portanto, métodos ativos de aprendizagem centrados no estudante precisam ser utilizados ${ }^{(44)}$.

Entre as mudanças mais urgentes para a promoção da sensibilidade moral dos enfermeiros está a necessidade de dar ênfase à "transformação pessoal". A sensibilidade moral de um indivíduo passa por um processo de desenvolvimento e mudança de forma contínua durante a produção da sua vida ${ }^{(24)}$. Nesse sentido, os estudantes já possuem um certo nível de sensibilidade moral quando iniciam o curso de enfermagem ${ }^{(24)}$. No entanto, espera-se que através de programas de formação eficazes e permanentes sobre ética, seja possível desenvolver sensibilidade moral, competência ética e habilidades práticas para conduzir à reflexão, discussão e enfrentamento prudente e responsável dos problemas éticos na prática. O enfermeiro que desenvolve sua sensibilidade moral será capaz de reconhecer mais problemas éticos na sua prática diária, e, por isso, precisa estar preparado para saber como resolver tais problemas, evitando o sofrimento moral.

Da mesma forma, "transformações contextuais" também são necessárias, pois um ambiente de trabalho que possibilita apoio ético aos enfermeiros através da promoção do diálogo interpessoal efetivo, com a possibilidade de partilha de sentimentos com colegas sobre uma determinada situação ética angustiante, fomenta a reflexão moral e, consequentemente, é capaz de promover a possibilidade de resolução dos problemas éticos ${ }^{(41)}$. Em resumo, ao se criar oportunidades para o enfermeiro refletir sobre os problemas éticos da prática com uma equipe multidisciplinar, é possível colaborar para a melhoria da qualidade do atendimento nos serviços de saúde ${ }^{(33)}$.

As mudanças nos processos de trabalho são mais complexas e difíceis de acontecer, por isso, é fundamental que ocorra a superação da fragmentação dos processos de trabalho, das relações entre os diferentes profissionais e do entendimento de saúde como simples ausência de doença ${ }^{(45)}$. Nesse contexto, o processo de trabalho precisa ser marcado pela cultura do trabalho vivo em ato que é aquele que está baseado na relação, e não na saúde como um produto com valor de troca, ou seja, o núcleo central da ação é a relação entre o profissional e o paciente. Quando o cuidado fica restrito a procedimentos, protocolos e prescrição de medicamentos ou exames, o trabalho transforma-se em morto $^{(46)}$.

A sensibilidade moral opera sobre um substrato moral muito complexo que envolve emoções, normas, valores e virtudes ${ }^{(47)}$, ou seja, opera principalmente sobre os "fatores pessoais". Nesse sentido, desenvolver a sensibilidade moral dos enfermeiros é uma tarefa desafiadora, se houver a compreensão de que a sensibilidade moral é muito mais do que apenas ter uma atitude compassiva - no sentido de partilhar o sofrimento do paciente - pois ela implica a construção de cenários imaginários em que o interesse e direitos do paciente entram em jogo, bem como as normas e valores que regem a prática profissional ${ }^{(47)}$.

O autor deste estudo procurou avaliar a maior parte da literatura existente, no entanto, reconhece algumas limitações neste processo, pois existem pesquisas publicadas em outros idiomas que não foram incluídas, bem como não se considerou uma diversidade de estudos que provavelmente existam em outras bases de indexação. Como o termo "sensibilidade moral" não é um descritor/MeSH, isto pode ter limitado a busca por estudos que utilizaram outros termos descritivos. A scoping review é apenas um dos métodos que podem ser utilizados para revisão da literatura e é provável que outros métodos pudessem chegar a resultados diferentes. 
Através da síntese dos resultados de pesquisas com relevância mundial, é possível estimular e promover a incorporação de evidências científicas na prática profissional, ou seja, permitir a transferência de conhecimento para os enfermeiros ao se destacar os fatores que influenciam a sensibilidade moral, conforme a literatura. Assim, para fazer a diferença na assistência à saúde, é imprescindível vincular o conhecimento oriundo de pesquisas ao da prática clínica.

\section{- CONCLUSÃo}

Este estudo identificou que os fatores que tem maior peso na formação/expressão da sensibilidade moral dos enfermeiros são os "fatores pessoais". Por outro lado, os "fatores contextuais" que influenciam a sensibilidade moral dependem principalmente da formação, da prática profissional, quando se estabelece um bom clima ético no ambiente de trabalho, com normas e regulamentos em coerência com os valores dos enfermeiros, bem como uma carga de trabalho adequada.

Vale destacar que a sensibilidade moral do enfermeiro não deve ser percebida apenas como ter consciência de como as suas ações afetam os pacientes, e sim, deve ir muito além, envolvendo a percepção e a interpretação do problema ético, assim como a capacidade de tomar decisões de forma justificável e prudente. Daí a necessidade de existirem estratégias de apoio que visem à promoção da sensibilidade moral ainda durante a formação, com metodologias ativas de ensino e aprendizagem, de modo a facilitar a incorporação de conhecimento ético. Da mesma forma, estratégias de apoio nos serviços de saúde devem ser implementadas, oportunizando momentos de reflexão e discussões entre os profissionais.

Portanto, os resultados desta revisão são úteis para futuras pesquisas na área, seja para a assistência, para o ensino e para a ação dos próprios enfermeiros, na medida em que permitem identificar os fatores que influenciam a construção/formação da sensibilidade moral desses profissionais, possibilitando-os a identificar e manejar de forma responsável e prudente os problemas éticos da sua prática. Novas pesquisas precisam ser realizadas, a fim de avaliar os fatores que tem maior peso na formação/expressão sensibilidade moral dos enfermeiros nos diferentes serviços de saúde.

\section{APOIO FINANCEIRO}

Bolsa de estudos concedida pela Coordenação de Aperfeiçoamento de Pessoal de Nível Superior (CAPES), pelo Programa Ciências sem Fronteiras, na modalidade de doutorado pleno no exterior, processo Bex: 2762-13-7.

\section{- REFERÊNCIAS}

1. Choe K, Park S, Yoo SY. Effects of constructivist teaching methods on bioethics education for nursing students: a quasi-experimental study. Nurse Educ Today. 2014;34(5):848-53.

2. Ahn SH, Yeom HA. Moral sensitivity and critical thinking disposition of nursing students in Korea. Int J Nurs Pract. 2014;20(5):482-9.

3. Milliken A. Nurse ethical sensitivity: An integrative review. Nurs Ethics. Epub ahead of print. 2016:1-26.

4. Lützén K, Evertzon M, Nordin C. Moral sensitivity in psychiatric practice. Nurs Ethics. 1997;4(6):472-82.

5. Lützén K, Dahlqvist V, Eriksson S, Norberg A. Developing the concept of moral sensitivity in health care practice. Nurs Ethics. 2006;13(2):187-96.

6. La Taille Y. Moral e ética: dimensões intelectuais e afetivas. Porto Alegre: Artmed; 2006.

7. Arksey H, O'Malley L. Scoping studies: towards a methodological framework. Int J Soc Res Methodol. 2005;8(1):19-32. 
8. Levac D, Colquhoun H, O'Brien KK. Scoping studies: advancing the methodology. Implementation Science. 2010;(5):69.

9. Grant MJ, Booth A. A typology of reviews: an analysis of 14 review types and associated methodologies. Health Info Libr J. 2009;26(2):91-108.

10. Armstrong R, Hall BJ, Doyle J, Waters E. Cochrane Update 'Scoping the scope' of a cochrane review. J Public Health. 2011;33(1):147-50.

11. Kim YS, Kang SW, Ahn JA. Moral sensitivity relating to the application of the code of ethics. Nurs Ethics. 2013;20(4):470-8.

12. Lützén K, Johansson A, Nordström G. Moral sensitivity: some differences between nurses and physicians. Nurs Ethics. 2000;7(6):520-30.

13. Lützén K, Blom T, Ewalds-Kvist B, Winch S. Moral stress, moral climate and moral sensitivity among psychiatric professionals. Nurs Ethics. 2010;17(2):213-24.

14. Han SS, Kim J, Kim YS, Ahn S. Validation of a korean version of the moral sensitivity questionnaire. Nurs Ethics. 2010;17(1):99-105.

15. Ersoy N, Göz F. The ethical sensitivity of nurses in Turkey. Nurs Ethics. 2001;8(4):299-312.

16. Bégat I, Ikeda N, Amemiya T, Emiko K, Iwasaki A, Severinsson E. Comparative study of perceptions of work environment and moral sensitivity among japanese and norwegian nurses. Nurs Health Sci. 2004;6(3):193-200.

17. Kim YS, Park JW, You MA, Seo YS, Han SS. Sensitivity to ethical issues confronted by korean hospital staff nurses. Nurs Ethics. 2005;12(6):595-605.

18. González-de Paz L, Kostov B, Sisó-Almirall A, Zabalegui-Yárnoz A. A Rasch analysis of nurses 'ethical sensitivity to the normsof the code of conduct. J. Clin. Nurs. 2012;21(19-20):2747-60.

19. Jaafarpour M, Khani A. Evaluation of the nurses' job satisfaction, and its association with their moral sensitivities and well-being. J Clin Diagn Res. 2012;6(10):1761-4.

20. Ertug N, Aktas D, Faydali S, Yalçin O. Ethical sensitivity and related factors of nurses working in the hospital settings. Acta Bioethica 2014;20(2):265-70.

21. Comrie RW. An analysis of undergraduate and graduate student nurses' moral sensitivity. Nurs Ethics. 2012;19(1):116-27.

22. Park M, Kjervik D, Crandell J, Oermann MH. The relationship of ethics education to moral sensitivity and moral reasoning skills of nursing students. Nurs Ethics. 2012;19(4):568-80.

23. Borhani F, Abbaszadeh A, Mohsenpour M. Nursing students' understanding of factors influencing ethical sensitivity: a qualitative study. Iranian J Nurs Midwifery Res. 2013;18(4):310-5.

24. Baykara ZG, Demir SG, Yaman S. The effect of ethics training on students recognizing ethical violations and developing moral sensitivity. Nurs Ethics. 2015;22(6):661-75.

25 Sahin SY, Iyigun E, Acikel C. Validity and reliability of a turkish version of the modified moral sensitivity questionnaire for student nurses. Ethics Behav. 2015;25(4):351-9.

26. Weaver K, Morse J, Mitcham C. Ethical sensitivity in professional practice: concept analysis. J Adv Nurs. 2008;62(5):607-18

27. You D, Maeda Y, Bebeau MJ. Gender differences in moral sensitivity: A Meta-Analysis. Ethics Behav. 2011;21(4):263-82.

28. Vanderheide R, Moss C, Lee S. Understanding moral habitability: a framework to enhance the quality of the clinical environment as a workplace. Contemp Nurse. 2013;45(1):101-13.

29. Jaeger SM. Teaching health care ethics: the importance of moral sensitivity for moral reasoning. Nursing 
Philosophy. 2001;2(2):131-42.

30. Weaver K, Morse JM. Pragmatic utility: using analytical questions to explore the concept of ethical sensitivity. Res Theory Nurs Pract. 2006;20(3):191-214.

31. Weaver K. Ethical sensitivity: state of knowledge and needs for further research. Nurs Ethics. 2007;14(2):14155.

32 Robichaux C. Developing ethical skills: from sensitivity to action. Crit Care Nurse. 2012;32(2):65-72.

33. Van Der Zande M, Baart A, Vosman F. Ethical sensitivity in practice: finding tacit moral knowing. J Adv Nurs. 2014;70(1):68-76.

34. Nortvedt P. Subjectivity and vulnerability: reflections on the foundation of ethical sensibility. Nurs Philos. 2003;4(3):222-30.

35. Melnyk BM, Fineout-Overholt E. Making the case for evidence-based practice. In: Melnyk BM, FineoutOverholt E. Evidence-based practice in nursing and healthcare: a guide to best practice. Philadelphia: Lippincot Williams \& Wilkins. 2005. p.3-24.

36. Dalla Nora CR, Zoboli ELCP, Vieira M. Problemas éticos vivenciados por enfermeiros na atenção primária à saúde: revisão integrativa da literatura. Rev. Gaúcha Enferm. 2015;36(1):112-21.

37. Ramos FR, Brehmer LC, Vargas MA, Trombetta AP, Silveira LR, Drago L. Ethical conflicts and the process of reflection in undergraduate nursing students in Brazil. Nurs Ethics. 2015;22(4):428-39.

38. Jackson D, Cleary M, Mannix J. Editorial: ethical sensitivity: shaping the everyday work environment. Contemp Nurse. 2013;44(1):2-4.

39. Shahriari M, Mohammadi E, Abbaszadeh A, Bahrami M. Nursing ethical values and definitions: a literature review. Iran J Nurs Midwifery Res. 2013;18(1):1-8.

40. Horton K, Tschudin V, Forget A. The value of nursing: a literature review. Nurs Ethics. 2007;14(6):716-40.

41. Lützén K, Ewalds-Kvist B. Moral distress and its interconnection with moral sensitivity and moral resilience: viewed from the philosophy of Viktor E. Frankl. J Bioeth Inq. 2013;10(3):317-24.

42. Schaefer R, Viera M. Competência ética como recurso de enfrentamento do sofrimento moral em enfermagem. Texto Contexto Enferm. 2015;24(2):563-73.

43. Scanlon C. Developing and maintaining ethical competence. HEC Forum. 1997;9(1):85-92.

44. Trobec I, Starcic AI. Developing nursing ethical competences online versus in the traditional classroom. Nurs Ethics. 2015;22(3):352-66.

45. Galavote HS, Zandonade E, Garcia ACP, Freitas PSS, Seidl H, Contarato PC, et al. O trabalho do enfermeiro na Atenção Primária à Saúde. Esc. Anna Nery. 2016;20(1):90-8.

46. Merhy EE. Saúde: a cartografia do trabalho vivo. São Paulo: Hucitec; 2007.

47. Semberoiz A. O momento ético: sensibilidade moral e educação médica. São Leopoldo: Editora UNISINOS; 2015. 